\title{
UNDERWATER TARGET DIRECTION OF ARRIVAL ESTIMATION BY SMALL ACOUSTIC SENSOR ARRAY BASED ON SPARSE BAYESIAN LEARNING
}

Wang Biao, Ph. D.

He Cheng, Ph. D.

School of Electronic and Information, Jiangsu University of Science and Technology, Zhenjiang,

Jiangsu Prov., China

\begin{abstract}
Assuming independently but identically distributed sources, the traditional DOA (direction of arrival) estimation method of underwater acoustic target normally has poor estimation performance and provides inaccurate estimation results. To solve this problem, a new high-accuracy DOA algorithm based on sparse Bayesian learning algorithm is proposed in terms of temporally correlated source vectors. In novel method, we regarded underwater acoustic source as a first-order auto-regressive process. And then we used the new algorithm of multi-vector SBL to reconstruct the signal spatial spectrum. Then we used the CS-MMV model to estimate the DOA. The experiment results have shown the novel algorithm has a higher spatial resolution and estimation accuracy than other DOA algorithms in the cases of less array element space and less snapshots.
\end{abstract}

Keywords: DOA, underwater acoustic signal processing, sparse Bayesian learning, temporally correlated source

\section{INTRODUCTION}

With the further development of ocean exploration, the DOA estimation based on micro-underwater location platform (such as UUV, unmanned underwater vehicle) $[1,2]$ has aroused great interest in this particular field or branch. Micro-platform is characterized by more flexibility and higher safety, which is the key to moving node in underwater sensor networks.

However, the traditional DOA technology, such as beamforming [3], the Capon's method [4] and the methods like MUSIC [5], can't be perfectly combined with the micro underwater positioning platform due to the restrictions on platform size and load. In recent years, DOA estimation based on compressed sensing theory[6] (CS) has been an active part in this particular field. In[7], a compressive beam-forming method for DOA estimation was proposed, and this approach does not be limited by Nyquist sampling theorem, but it is susceptible to signal correlation. In [8], a kind of spatial compressive sampling for underwater acoustic target is applied to DOA estimation, the method increases the DOA estimation accuracy in the cases of less snapshots and less array elements. Through a further research of CS, the signal reconstruction by compressive measurements has been studied from the perspective of Bayesian. In [9], the author proposed Bayesian compressive sensing for DOA determination. By adopting the idea of multitasking learning, it avoids the array output covariance matrix designing and reduces the computational complexity. In[10], an efficient DOA estimation approach was proposed via sparse Bayesian learning, the present approach solves the sparse DOA estimation problem in real domain by making good use of the special geometry of the uniform linear array. This method provides higher spatial accuracy and lower computational complexity. However, the algorithm mentioned above is based on the hypothesis that the signal sources are mutually independent, that is to say, equally distributed. In this case, the spatial structure of the source 
signal is solely taken into account, without including the time-correlation properties of the signal source. According to current scientific knowledge, a wide range of signals, including biomedical signal, voice signal, geophysical and underwater acoustic signal, are, to a limited extent, timedependent. When the underwater target may be move quickly during the time of continuous observation, the continuous snapshots would have correlation between them, which will degrade the estimation precision. If the priori knowledge of that signal is applied to the DOA estimation model, a more effective estimation will be obtained. Then we propose a new method based on SBL[11,12,13] for underwater acoustic target, which can solve the problem to get more performance. Furthermore, considering MacKay's fixed-point method[14], a fast DOA estimation method based on sparse Bayesian learning is proposed.

In order to utilize the correlation of source data, the algorithm assumes the correlation as a first-order autoregressive time process, which the structure sparsity of signal can be fully applied to DOA estimation model. In addition, the $C S-M M V$ model[15] is established to model the DOA with the correlation. Finally, Muti-vectors Sparse Bayesian Learning has been applied to acquired the signal space spectrum by utilizing the structural sparsity. The simulation results have shown that the novel algorithm that we proposed has a higher spatial resolution than many algorithms in the cases of less array elements and less snapshots, which fits well into the micro-platform, such as unmanned underwater vehicle.

\section{PROBLEM STATEMENT}

In this paper, we consider $M$ narrow-band far field static sources with unknown DOAs $\left\{\theta_{1}, \theta_{2}, \ldots, \theta_{M}\right\}$ imping on a uniform linear array (ULA), consisting of $N$ $(N>M)$ identical sensors with inter-sensor spacing $d$ (half-wavelength), which is described in Fig.1. The received complex signal data is represented as:

$$
y(t)=A(\theta) x(t)+e(t) \quad t \in\left\{t_{1}, \ldots, t_{L}\right\}
$$

where $A(\theta)=\left[a\left(\theta_{1}\right), a\left(\theta_{2}\right), \ldots, a\left(\theta_{M}\right)\right]$ is the matrix of the steering vectors whose $m$-th column is given by $a\left(\theta_{m}\right)$ $\theta=\left[\theta_{1}, \ldots, \theta_{M}\right]$ is the vector of unknown source location, $y(t)$ is the $t-t h$ observed snapshot, $x(t)$ is the $t-t h$ snapshots of the unknown source arriving signals, $e(t)$ is the $t-t h$ snapshot of the additive spatial white noise, $L$ represents the number of snapshots. Gathering the multiple snapshots the model (1) can be rewritten as:

$$
Y=A(\theta) X+E
$$

where $Y \triangleq\left[y_{.1}, y_{.2}, \ldots, y_{. L}\right] \in \mathbb{R}^{N \times L}$ is the observation matrix with $L$ measurement vectors. $X \triangleq\left[x_{1}, x_{2}, \ldots, x_{L}\right] \in \mathbb{R}^{M \times}$ is the unknown source matrix. $E \triangleq\left[e_{.1}, e_{.2}, \ldots, e_{. L}\right] \in \mathbb{R}^{N \times L}$ is the spatial white noise with zero mean and unknown variance $\sigma^{2}$.

To formulate the DOA estimation problem into SBL approach, uniformly we divide underwater acoustic target angle space into $K \gg N>M$ possible angles of arrival and construct a redundant matrix of $K$ atoms relating to the array responses of the respective angles of arrival (Fig.2). Then the transposed matrix $A(\theta)$ can be expressed as:

$$
\Phi=\left[\phi\left(\theta_{1}\right), \phi\left(\theta_{2}\right), \ldots, \phi\left(\theta_{K}\right)\right]
$$

Then the model (2) can be reformulated as:

$$
Y=\Phi \tilde{X}+E
$$

So, the estimation problem turns out to be that of recovering the sparse signal vectors $\tilde{X} \in \mathbb{C}^{K \times 1}$. Since the problem is linear against to the unknown $\tilde{X}$, and the solution is sparse in the spatial domain, the SBL theory can be properly applied.

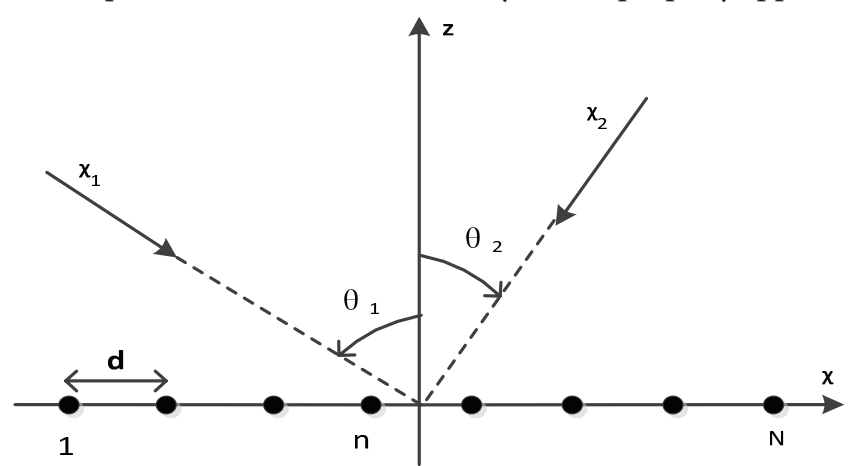

Fig.1 Sketch of the reference scenario: uniform linear array and impinging signals.

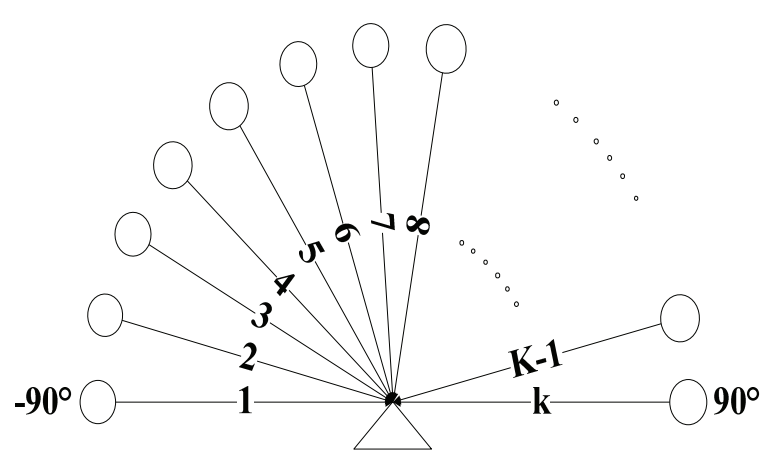

Fig.2 The angle space sparsity model of underwater acoustic target 


\section{UNDERWATER ACOUSTIC TARGET DOA ESTIMATION METHOD BASE ON SPARSE BAYESIAN LEANING}

Usually, we make the hypothesis that the multiple measurement vectors have the same sparsity structure, but unknown. So the multiple measurement vectors could be stacked based on space structure. The support of each source vector is slowly time-varying, we can utilize the concatenate of MMV models to approximate this scenario. In fact, the underwater acoustic target signal is a kind of signal with temporal structure, so each signal source satisfies an AR(1) model given by:

$$
\tilde{X}_{i, j+1}=\beta \tilde{X}_{i, j}+\sqrt{1-\beta} n_{i, j}, i=1, \ldots, K ; j=1, \ldots, L
$$

where $\beta \in(-1,1)$ is the AR coefficient, and we assume $n_{i, k} \sim N\left(0, \gamma_{i}\right)$ and $\tilde{X}_{i, k} \sim N\left(0, \gamma_{i}\right)$. A value of zero results $\gamma_{i}$ in a row with zero entries promoting sparsity. Obviously, if , the MMV model becomes the one with i.i.d sources. If $\beta= \pm 1$, the multiple measurement vector (MMV) model is equivalent to the single measurement vector (SMV) model in terms of recovery performance.

With the AR(1) modeling assumption, the joint distribution of $\tilde{X}_{i .}=\left[\tilde{X}_{i 1}, \tilde{X}_{i 2}, \ldots, \tilde{X}_{i L}\right]$ is given by

$$
\rho\left(\tilde{X}_{i,} ; \gamma_{i}, B_{i}\right) \sim N\left(0, \gamma_{i} B_{i}\right), \quad i=1,2, \ldots, K
$$

where $B$ is defined as:

$$
B \equiv\left[\begin{array}{cccc}
1 & \beta & \cdots & \beta^{L-1} \\
\beta & 1 & \cdots & \beta^{L-2} \\
\vdots & \vdots & \ddots & \vdots \\
\beta^{L-1} & \beta^{L-2} & \cdots & 1
\end{array}\right]
$$

where $\gamma_{i}$ is a nonnegative hyper-parameter controlling the row sparsity of $\tilde{X}$ as in the basic SBL. When $\gamma_{i}=0$, the associated $\tilde{X}_{i}$ becomes zero. $B_{i}$ is a positive definite matrix that captures the the correlation structure of $\tilde{X}$ and needs to be estimated. Note that in contrast to the original SBL framework, the BSBL framework models is the temporal structures of sources in the prior density via the matrices $B_{j} \quad(i=1, \ldots, M)$.The covariance matrix in the density of $X$ is $\gamma_{i} B_{i}$.

Assuming $y=\operatorname{vec}\left(Y^{T}\right) \in R^{N L \times I}, D=\Phi \otimes I_{L}$, $x=\operatorname{vec}\left(\tilde{X}^{T}\right) \in R^{M L \times I}, e=\operatorname{vec}\left(E^{T}\right)$, we can propose the model of block-spare Bayesian learning framework of DOA as follows:

$$
y=D x+e
$$

Assuming that the noise $e$ is independent and has the Gaussian distribution as:

$$
\rho\left(e_{i}\right) \sim N(0, \lambda)
$$

where $e_{i}$ is $i-t h$ element in $e, \lambda$ is variance, for the block model (8), the Gaussian likelihood is:

$$
\rho(y \mid x ; \lambda) \sim N_{y \mid x}(D x, \lambda I)
$$

The prior for $\tilde{X}$ is given by

$$
\rho\left(x ; \gamma_{i}, B_{i}, \forall i\right) \sim N_{x}\left(0, \Sigma_{0}\right)
$$

where $\Sigma_{0}$ is block diagonal matrix given by (12) with many diagonal block matrices being zeros.

$$
\Sigma_{0}=\left[\begin{array}{ccc}
\gamma_{1} B_{1} & & \\
& \ddots & \\
& & \gamma_{K} B_{K}
\end{array}\right]
$$

Using the Bayesian rule, the posterior distribution of $x$ can be expressed as a multivariate Gaussian distribution:

$$
\rho\left(x \mid y ; \lambda, \gamma_{i}, B_{i}, \forall_{i}\right)=N_{x}\left(u_{x}, \Sigma_{x}\right)
$$

with the mean vector

$$
u_{x}=\frac{1}{\lambda} \Sigma_{x} D^{T} y
$$

and the covariance matrix

$$
\begin{aligned}
\Sigma_{x} & =\left(\Sigma_{0}^{-1}+\frac{1}{\lambda} D^{T} D\right)^{-1} \\
& =\Sigma_{0}-\Sigma_{0} D^{T}\left(\lambda I+D \Sigma_{0} D^{T}\right)^{-1} D \Sigma_{0}
\end{aligned}
$$

Then the DOA problem is shifted to estimate all the hyper-parameter vectors $\lambda, \gamma_{i}, B_{i}, \forall_{i}$, when given all the hyper-parameters, we can obtain the maximum a posterior probability estimate of $x$.

$$
\begin{aligned}
x^{*} \triangleq u_{x} & =\left(\lambda \Sigma_{0}^{-1}+D^{T} D\right)^{-1} D^{T} y \\
& =\Sigma_{0} D^{T}\left(\lambda I+D \Sigma_{0} D^{T}\right)^{-1} y
\end{aligned}
$$

\section{HYPER-PARAMETERS ESTIMATION}

The ways of learing the matrices have different algorithms. In this section, we will discuss the learning of these hyper-parameters.

To find the hyper-parameters $\Theta=\left\{\lambda, \gamma_{1}, \ldots, \gamma_{M}, B\right\}$, Maximization $\rho(y ; \Theta)$ with respect to $\Theta$ can be done via Expectation-Maximization(EM) method. This is equivalent to minimizing $-\log \rho(y ; \Theta)$, yielding the effective cost function: 


$$
\begin{aligned}
L(\Theta) & \triangleq-2 \log \int \rho(y \mid x ; \lambda) \rho\left(x, \gamma_{i}, B_{i}, \forall_{i}\right) d x \\
& =\log \left|\Sigma_{y}\right|+y^{T} \Sigma_{y}{ }^{-1} y
\end{aligned}
$$

where $\Sigma_{y} \triangleq \lambda I+D \Sigma_{0} D^{T}$.

There are two ways to minimize $L(\Theta)$ with respect to $\gamma$. First, we can minimize this expression over $\gamma$ using EM method. Then we can obtain the learning rule for $\gamma$ :

$$
\gamma_{i} \leftarrow \frac{\operatorname{Tr}\left[B^{-1}\left(\sum_{x}^{i}+u_{x}^{i}\left(u_{x}^{i}\right)^{T}\right)\right]}{L}, \quad i=1, \ldots, K
$$

Second, at the expense of proven convergence, we may instead optimize (17) by taking the derivative with respect to $\gamma$, then letting the derivative equal to zero and following MacKay's fixed point approach, we have:

$$
\gamma_{i} \leftarrow \frac{\left(u_{x}^{i}\right)^{T} B^{-1} u_{x}^{i}}{L-\operatorname{Tr}\left(\sum_{x}^{i} B^{-1}\right) / \gamma_{i}}, \quad i=1, \ldots, K
$$

For the other two hyper-parameters $B, \lambda$, we also use EM method to solve the learning rule. They are given by:

$$
\begin{gathered}
B \leftarrow \frac{1}{K} \sum_{i=1}^{K} \frac{\sum_{x}^{i}+u_{x}^{i}\left(u_{x}^{i}\right)^{T}}{r_{i}} \\
\lambda \leftarrow \frac{\left\|y-D u_{x}\right\|_{2}^{2}+\lambda\left[K \times L-\operatorname{Tr}\left(\sum_{x} \Sigma_{0}^{-1}\right)\right]}{M \times L}
\end{gathered}
$$

For convenience, we first list the conclusions of MSBL(Multiple SBL) algorithm.

$$
\begin{aligned}
& \Xi_{x}=\left(\Gamma^{-1}+\frac{1}{\lambda} \Phi^{T} \Phi\right)^{-1} \\
& \tilde{X}=\Gamma \Phi^{T}\left(\lambda I+\Phi \Gamma \Phi^{T}\right)^{-1} Y
\end{aligned}
$$

where $\Gamma$ is defined as $\operatorname{diag}(\gamma)$.

According to the conclusions of MSBL algorithm, we can find that the matrix operations have lower dimension in this algorithm. So we attempt to achieve similar complexity by adopting the following approximation. And in the following section, we can use the following approximation to simplify the learning rule of hyper-parameters.

$$
\begin{aligned}
\left(\lambda I_{M L}+D \Sigma_{0} D^{T}\right)^{-1} & =\left(\lambda I_{M L}+\left(\Phi \Gamma \Phi^{T}\right) \otimes B\right)^{-1} \\
& \approx\left(\lambda I_{M L}+\Phi \Gamma \Phi^{T}\right)^{-1} \otimes B^{-1}
\end{aligned}
$$

Using the same approximation (23) and following the equation (15), the $u_{x}$ in (18) can be expressed as

$$
\begin{aligned}
u_{x} & =\Sigma_{0} \Phi^{T}\left(\lambda I+\Phi \Sigma_{0} \Phi^{T}\right)^{-1} y \\
& =(\Gamma \otimes B) \Phi^{T}\left[\lambda I+\Phi(\Gamma \otimes B) \Phi^{T}\right]^{-1} \operatorname{vec}\left(Y^{T}\right) \\
& \approx\left(\Gamma \Phi^{T} \otimes\right)\left[\left(\lambda I+\Phi \Gamma \Phi^{T}\right)^{-1} \otimes B^{-1}\right] \operatorname{vec}\left(Y^{T}\right) \\
& =\left[\Gamma \Phi^{T}\left(\lambda I+\Phi \Gamma \Phi^{T}\right)^{-1}\right] \otimes I \cdot \operatorname{vec}\left(Y^{T}\right) \\
& =\operatorname{vec}\left(Y^{T}\left(\lambda I+\Phi \Gamma \Phi^{T}\right)^{-1} \Phi \Gamma\right) \\
& =\operatorname{vec}\left(\tilde{X}^{T}\right)
\end{aligned}
$$

Then we can transform the $\gamma_{i}$ learning rule (18) to the following form according to the above analysis.

$$
\gamma_{i} \leftarrow \frac{1}{L} \tilde{X}_{i .} B^{-1} \tilde{X}_{i .}^{T}+\left(\Xi_{x}\right)_{i i}
$$

Similarly, we can transform the $\gamma_{i}$ learning rule (19) to the following form:

$$
\gamma_{i} \leftarrow \frac{\tilde{X}_{i .} B^{-1} \tilde{X}_{i .}^{T}}{L\left(1-\Xi_{i i} / \gamma_{i}\right)}, \quad \forall i
$$

Based on the approximation (25), we can simplify the $B$ learning rule (20) to the following form.

$$
B \leftarrow \frac{1}{K} \sum_{i=1}^{K} \frac{\left(\Xi_{x}\right)_{i i} B+\tilde{X}_{i .}^{T} \tilde{X}_{i .}}{\gamma_{i}}
$$

To increase the robustness, we use the rule below:

$$
B \leftarrow \tilde{B} /\|\tilde{B}\|_{F}, \tilde{B}=\sum_{i=1}^{K} \frac{\tilde{X}_{i .}^{T} \tilde{X}_{i .}}{\gamma_{i}}+\eta I
$$

where $\eta$ is a positive scalar. This regularization item in $\tilde{B}$ ensures that $B$ is positive definite.

Similarly, we simplify the $\lambda$ learning rule (21) as follows:

$$
\begin{aligned}
\lambda & \leftarrow \frac{\left\|y-D u_{x}\right\|_{2}^{2}+\lambda\left[K \times L-\operatorname{Tr}\left(\Sigma_{x} \Sigma_{0}^{-1}\right)\right]}{M \times L} \\
& =\frac{\left.\left\|y-D u_{x}\right\|_{2}^{2}+\lambda \operatorname{Tr}\left(\Sigma_{0} D^{T} \Sigma_{y}^{-1} D\right)\right]}{M \times L} \\
& \approx \frac{1}{M \times L}\|Y-\Phi \tilde{X}\|_{F}^{2}+\frac{\lambda}{M \times L} \operatorname{Tr}\left[(\Gamma \otimes B)\left(\Phi^{T} \otimes I\right)\right. \\
& =\frac{\left.\left(\left(\lambda I+\Phi \Gamma \Phi^{T}\right)^{-1} \otimes B^{-1}\right)(\Phi \otimes I)\right]}{M \times L}\|Y-\Phi \tilde{X}\|_{F}^{2}+\frac{\lambda}{M} \operatorname{Tr}\left[\Phi \Gamma \Phi^{T}\left(\lambda I+\Phi \Gamma \Phi^{T}\right)^{-1}\right]
\end{aligned}
$$




\begin{tabular}{|c|c|c|c|}
\hline Symbol & implication & Symbol & implication \\
\hline$M$ & $\begin{array}{l}\text { the number of narrow-band far field } \\
\text { static sources }\end{array}$ & $\gamma_{i}$ & a nonnegative hyper-parameter \\
\hline$N$ & the number of sensors & $B_{i}$ & a positive definite matrix \\
\hline$d$ & inter-sensor spacing & $y$ & $\operatorname{vec}\left(Y^{T}\right)$ \\
\hline$\theta$ & the vector of unknown source location & $x$ & $\operatorname{vec}\left(\tilde{X}^{T}\right)$ \\
\hline$A(\theta)$ & the matrix of the steering vectors & $e$ & $\operatorname{vec}\left(E^{T}\right)$ \\
\hline$y(t)$ & the $t$-th observed snapshot & $\Sigma_{0}$ & block diagonal matrix \\
\hline$x(t)$ & $\begin{array}{l}\text { the } t-t h \text { snapshot of the unknown } \\
\text { arriving signals }\end{array}$ & $u_{x}$ & the mean vector \\
\hline$e(t)$ & $\begin{array}{l}\text { the } t-t h \text { snapshot of the additive } \\
\text { spatial white noise }\end{array}$ & $\Sigma_{x}$ & the covariance matrix \\
\hline$L$ & the number of snapshots & $x^{*}$ & $\begin{array}{l}\text { the maximum a posterior probability estimate of } \\
\qquad x\end{array}$ \\
\hline$Y$ & the observation matrix & $\Theta$ & the hyper-parameters \\
\hline$X$ & the unknown source matrix & $L(\Theta)$ & the effective cost function \\
\hline$E$ & $\begin{array}{l}\text { the number of discreting the } \\
\text { underwater acoustic target signal space }\end{array}$ & $\Xi_{x}$ & the conclusions of MSBL(Multiple SBL) algorithm \\
\hline$K$ & $\begin{array}{l}\text { the number of discreting the } \\
\text { underwater acoustic target signal space }\end{array}$ & $\Gamma$ & $\operatorname{diag}(\gamma)$ \\
\hline$\tilde{X}$ & the sparse signal vectors & $\eta$ & a positive scalar \\
\hline$\beta$ & the AR coefficient & & \\
\hline
\end{tabular}

According to the above theory described, the new algorithm process aims at obtaining the maximum posterior probability estimation of signal sources by solving the hyper-parameters' value, then it can get the estimation of target azimuth. According to the derivation of the above theories, we can summarize the algorithm implementation steps as table 2 .

\section{SIMULATION RESULTS}

In this section, several simulation schemes for the underwater acoustic target DOA estimation algorithm are proposed for a detailed discussion.

We consider a uniform linear array of $M=20$ identical sensors separated by half a wavelength of narrowband underwater acoustic signals. Three narrowband underwater acoustic target signals in the far-field impinge on this array from different DOAs. Here we simulate three sources at $-20^{\circ}$, $-18^{\circ}$ and $20^{\circ}$ with $S N R$ of $10 \mathrm{~dB}$, and all the signals were $\mathrm{AR}(1)$ processes with the common AR coefficient $\beta=0.8$. The number of snapshots is $L=100$, squeezed number is $M=10$ and the number of potential locations is $K=361$.

\section{THE FEASIBILITY ANALYSIS OF OUR PROPOSED ALGORITHM}

Fig. 3 shows the simulation results of spatial spectrum, where there are more than one target. Comparing them we can conclude that Capon's method has better performance when array elements are 40 . Our proposed methods can always localize the sources. From the Fig.3(c) and Fig.3(d), the 
b)

\section{Initialization}

1. Set $\lambda:=1, \gamma:=1$.

2. Hyper-meter $B$ is setted to the $M$ order unit array with the main diagonal' value of 1 , where $M$ is the number of single sources.

Repeat

3. Compute the posterior moments $u_{x}$ and $\Sigma_{x}$ using (14) and (15).

4. Update the learning rule of $\lambda, \gamma, B$ using EM rule or the faster fixed point rule with respect to $\gamma$.

5. while three hyper-meters $\lambda, \gamma, B$ convergence to a fixed point.

6. Calculate the value of $u_{x}^{*} \triangleq E[x \mid y ; \Theta]$, given that $\gamma$ is sparse, the resultant estimator $u_{x}^{*}$ will necessarily be row sparse.

7. If given the value of $u_{x}^{*}$, then we can find out the value of $x^{*}$. Based on the maximum posterior probability $x^{*}$ of underwater acoustic signal sourcex, we can restore the underwater acoustic target source signal and complete the underwater acoustic target DOA estimation.

traditional two methods failed to resolve the closely spaced $\left(5^{\circ}\right.$ separation) sources due to the Rayleigh limit, regardless of the array element spacing. However, our algorithms are still having a very high resolution, with two sharp spectral peaks. In Fig.3(c) and Fig.3(d), the number of sources was increased to 4 . When the array spacing $\mathrm{d}$ is larger enough, Capon's method and Beam-forming can approximately locate the sources. But these two traditional algorithms have strong bias when the array spacing is decreased to 0.2. From fig(a) to fig(f), the proposed methods always get the sharper peaks and distinguishes the sources clearly. We can demonstrate that the proposed method gets the underwater acoustic target DOA estimation is feasible.

a)

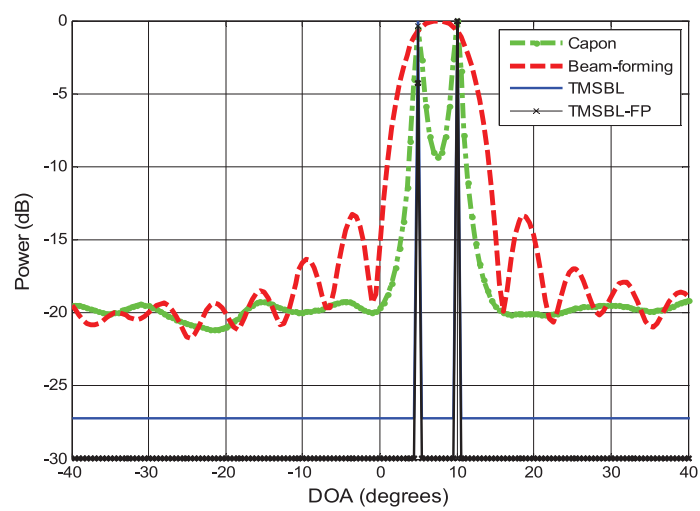

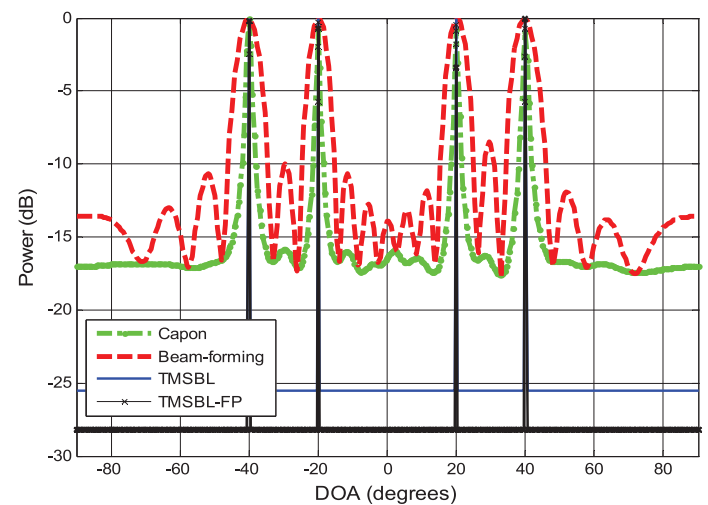

c)

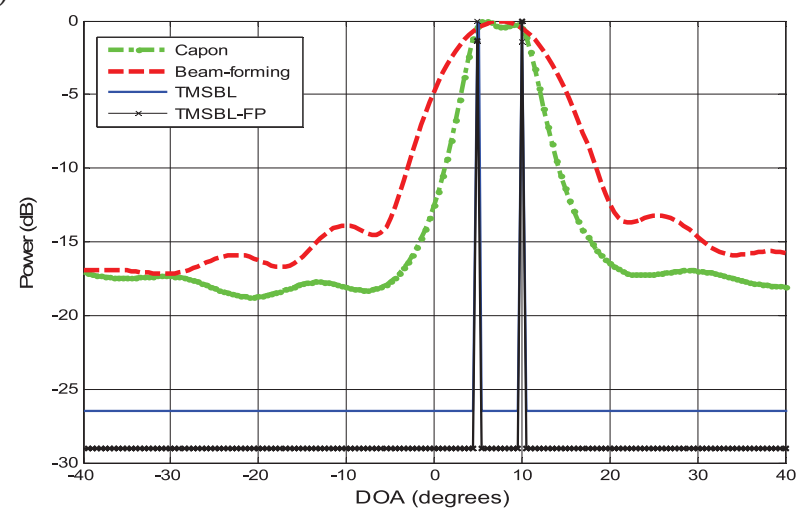

d)

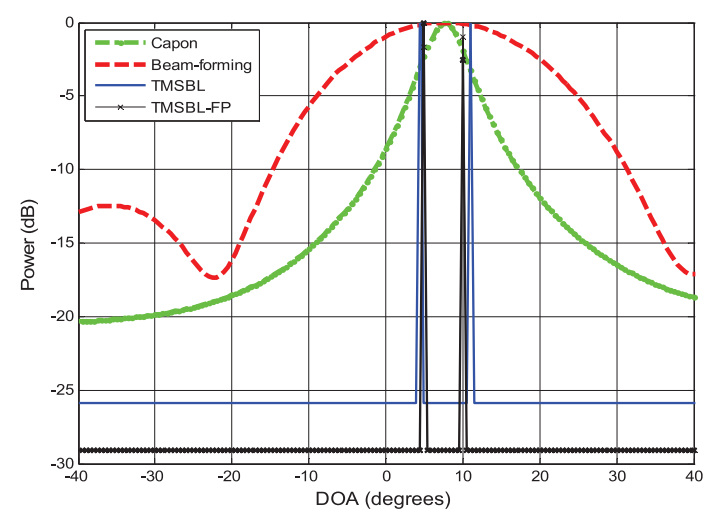

e)

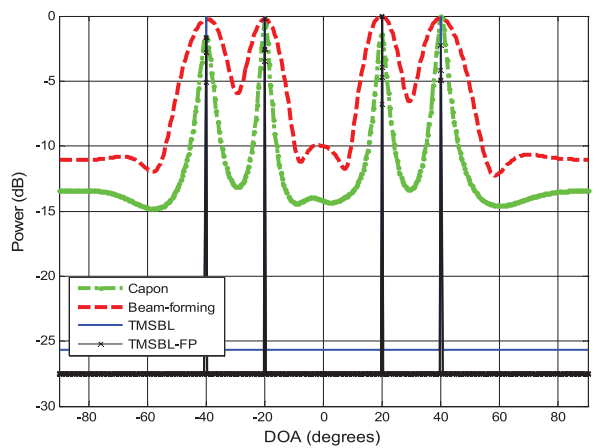

(e) 
f)

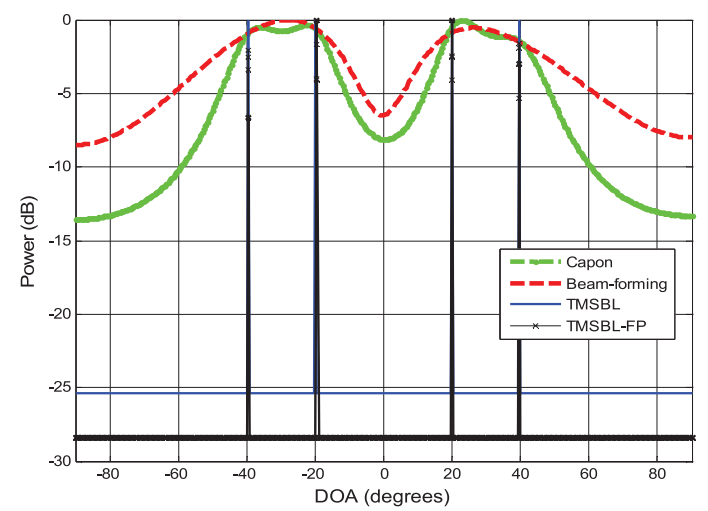

Fig. 3 Spatial spectrum obtained by different algorithms, $S N R=10 d B, L=100$ (a)Temporally correlated sources at $5^{\circ}$ and $10^{\circ}$, array spacing $d=0.5$, array elements $N=40$.(b)Four temporally correlated sources, array spacing $d=0.5$, array elements $N=40$. (c)Temporally correlated sources at $5^{\circ}$ and $10^{\circ}$, array spacing $d=0.5$, array elements $N=20$. (d) Temporally correlated sources at $5^{\circ}$ and $10^{\circ}$, array spacing $d=0.2$, array elements $N=20$. (e) Four temporally correlated sources, array spacing $d=0.5$, array elements $N=20$. $(f)$ Four

temporally correlated sources, array spacing $d=0.2$, array elements $N=20$.

\section{RECOVERY ABILITY FOR DIFFERENT ALGORITHMS AT DIFFERENT NOISE LEVELS}

The RMSE with SNR simulation results of the proposed algorithms(TMSBL and TMSBL-FP) were showed in Fig.4 comparing with two traditional algorithms and CS algorithm, Capon's ,beam-forming and CS-L0 method In Fig.4(a), the snapshots number is fixed at 100, and in Fig.4(b), the snapshots number is fixed at 200. The results in Fig.4(a)-(b) show that TMSBL-FP algorithm achieve super resolution at the lowest $S N R$, and the TMSBL algorithm can also get better performance at the same SNR. Their RMSE are also smaller than Capon's method and beam-forming at any different SNR. Comparing with the CS-L0 algorithm based on CS. The simulation results show that the proposed algorithm also has a better estimation performance than that of [8]. But the algorithm's performance doesn't have more obvious advantages in the estimation precision than those of the algorithm of [8] if the signal is not the type of 'time-correlation structure sparsity'.

a)

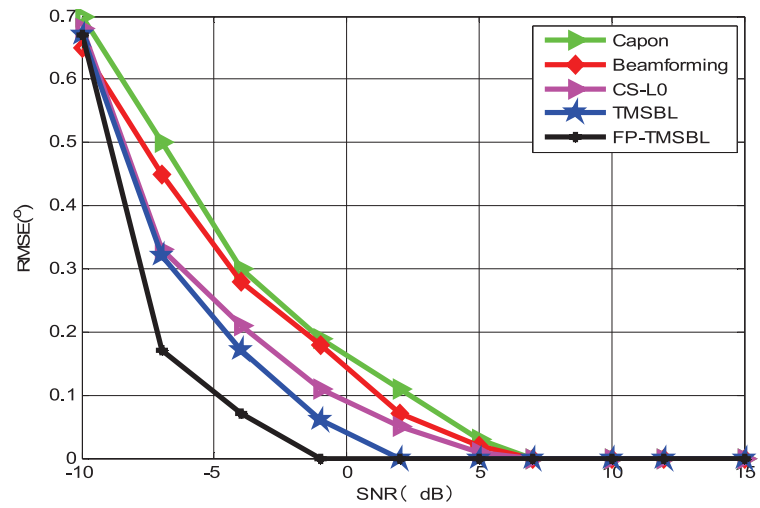

b)

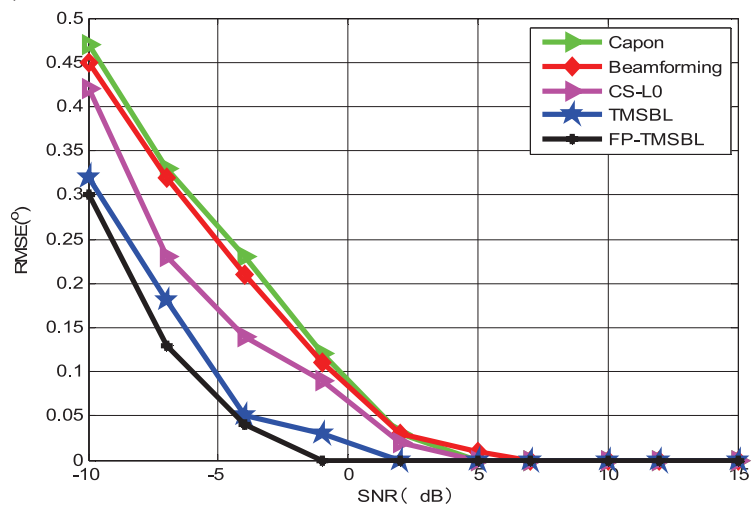

Fig.4 The RMSE with SNR simulation results of the proposed algorithms(TMSBL and TMSBL-FP)

\section{THE COMPUTATION TIME FOR DIFFERENT ALGORITHMS}

Tab. 3 The time of these algorithms for achieving the DOA estimation

\begin{tabular}{|c|c|c|c|c|}
\hline Time(s) & 0.063467427 & 0.063507871 & 1.574971 & 0.062059304 \\
\hline Algorithm & Capon & $\begin{array}{c}\text { Beam- } \\
\text { forming }\end{array}$ & TMSBL & TMSBL-FP \\
\hline
\end{tabular}

We compare the computation time for different algorithms. As can be seen in tab. 3, Capon's method and Beam-forming algorithm used nearly the same time as TMSBL-FP algorithm, and TMSBL-FP algorithm turns out to be the slowest. However, it is important to indicate that the performance of Capon's method and Beam-forming are much worse than algorithms of TMSBL or TMSBL-FP if the source is temporally correlated.

\section{CONCLUSIONS}

In this paper, it is shown that existing DOA estimation algorithms have poor performance. To solve these problems, a novel method and the faster algorithm of DOA estimation is proposed to realize the real time. The simulation experiment results show that the proposed novel algorithm has more superior performance than many other algorithms considering the data correlation as the structured sparsity. This method is suitable for underwater acoustic target DOA estimation in micro platforms (i.e. UUV) due to the fact that the method needs less array elements and less snapshots to localize the quick movement underwater target.

\section{ACKNOWLEDGMENT}

The study was sponsored by the National Natural Science Foundation of China (Grant No.11574120, No.U1636117), the Natural Science Foundation of Jiangsu Province (Grant 
No.BK20161359), Project of the Priority Academic Program Development of Jiangsu Higher Education Institutions, the Open Project Program of the Key Laboratory of Underwater Acoustic Signal Processing, Ministry of Education, China (No.UASP1503), the Fundamental Research Funds for the Central Universities (2242013K30010) and Qing Lan Project.

\section{REFERENCES}

1. Daegil Park, Jaehoon Jung, Kyungmin Kwak,Wan Kyun Chung, Jinhyun kim.:3D underwater localization using EM waves attenuation for UUV docking, IEEE Underwater Technology (UT),pp.1-4,2017.

2. Mohd Shahrieel Mohd Aras, Muhammad Nizam Kamarudin,et al.:Analysis of integrated sensors for unmanned underwater vehicle application,2016 IEEE International Conference on Underwater System Technology: Theory and Applications (USYS),pp.224-229,2016.

3. Despoina Pavlidi,Symeon Delikaris-Manias,Ville Pulkki,et al.:3D DOA estimation of multiple sound sources based on spatially constrained beamforming driven by intensity vectors, 2016 IEEE International Conference on Acoustics, Speech and Signal Processing (ICASSP),pp.96-100,May 2016.

4. Shigeaki Okumura, Hirofumi Taki,Toru Sato.:Stabilization techniques for high resolution ultrasound imaging using beam space Capon method,2015 IEEE International Conference on Acoustics, Speech and Signal Processing (ICASSP),pp.892-896,2015.

5. Zaidao Wen, Biao Hou, Licheng Jiao .:Joint Sparse Recovery With Semi-supervised MUSIC,IEEE Signal Processing Letters , vol.24,pp.629-633,2017.

6. Chun-Yu Kang, Qian-Yan Li,Yi-Ming Jiao,et al.: Direction of arrival estimation and signal recovery for underwater target based on compressed sensing, International Congress on Image and Signal Processing(CISP), pp.1277-1282,2015.

7. Md Mashud Hyder, Kaushik Mahata.: Direction-of-Arrival Estimation Using a Mixed $\bigotimes 20$ Norm Approximation, IEEE Transactions on signal Proc, vol.58, no 9, pp. 4646-4655, 2010.

8. WANG, B., Li, C. and Dai, Y. W.: DOA estimation method based on spatial compressive sampling for underwater acoustic target, Acta Armamentarii, vol.34, no 11, pp. 14791483, 2013.

9. Carlin M, Rocca P, Oliveri G, et al.: Directions-of-arrival estimation through Bayesian compressive sensing strategies, IEEE Transactions on Antennas and Propagation, vol.61, no7, pp. 3828-3838, 2013.
10. Sun, L.,Wang, H. L., Xu, G. J. et al.: Efficient Direction-ofarrival Estimation via Sparse Bayesian Learning, Jounal of Electronics \& Information Technology, vol.35, no5, pp.1196-1201,2013.

11. Anup Das, Terrence J. Sejnowski.: Narrowband and Wideband Off-Grid Direction-of-Arrival Estimation via Sparse Bayesian Learning,IEEE Journal of Oceanic Engineering,pp.1-11,2017.

12. Peter Gerstoft, Christoph F. Mecklenbräuke.: Wideband Sparse Bayesian Learning for DOA Estimation from multiple snapshots, IEEE Sensor Array and Multichannel Signal Processing Workshop (SAM),pp.1-5,2016.

13. Peter Gerstoft,Christoph F. Mecklenbräuke,et al.: Multisnapshot Sparse Bayesian Learning for DOA, IEEE Signal Processing Letters, vol. 23,pp.1469-1473,2016.

14. MacKay, D.:Bayesian Interpolation Neural Computation, vol.4, no 3, pp.415-447, May. 1992.

15. Wang, F. S., Zhang, L. R., Zhou, Y., et al.: Multiple Measurement Vectors for Compressed Sensing: Model and Algorithms Analysis, Signal Processing, vol.28, no 6, pp.785792, 2012.

\section{CONTACT WITH THE AUTHOR}

Wang Biao, Ph.D.

He Cheng, Ph.D.

Faculty of School of Electronic and Information, Jiangsu University of Science and Technology Zhenjiang, Jiangsu, 212003, China e-mail:wangbiao@just.edu.cn CHINA 